\title{
COLORFUL COVERINGS OF POLYTOPES AND PIERCING NUMBERS OF COLORFUL $d$-INTERVALS
}

\author{
FLORIAN FRICK AND SHIRA ZERBIB
}

\begin{abstract}
We prove a common strengthening of Bárány's colorful Carathéodory theorem and the KKMS theorem. In fact, our main result is a colorful polytopal KKMS theorem, which extends a colorful KKMS theorem due to Shih and Lee [Math. Ann. 296 (1993), no. 1, 35-61] as well as a polytopal KKMS theorem due to Komiya [Econ. Theory 4 (1994), no. 3, 463-466]. The (seemingly unrelated) colorful Carathéodory theorem is a special case as well. We apply our theorem to establish an upper bound on the piercing number of colorful $d$-interval hypergraphs, extending earlier results of Tardos [Combinatorica 15 (1995), no. 1, 123-134] and Kaiser [Discrete Comput. Geom. 18 (1997), no. 2, 195-203].
\end{abstract}

MSC codes: 55M20, 52B11, 05B40, 52A35

\section{INTRODUCTION}

The KKM theorem of Knaster, Kuratowski, and Mazurkiewicz [11] is a set covering variant of Brouwer's fixed point theorem. It states that for any covering of the $k$-simplex $\Delta_{k}$ on vertex set $[k+1]$ with closed sets $A_{1}, \ldots, A_{k+1}$ such that the face spanned by vertices in $S$ is contained in $\bigcup_{i \in S} A_{i}$ for every $S \subset[k+1]$, the intersection $\bigcap_{i \in[k+1]} A_{i}$ is nonempty.

The KKM theorem has inspired many extensions and variants, some of which we will briefly survey in Section 2. Important strengthenings include a colorful extension of the KKM theorem due to Gale [9] that deals with $k+1$ possibly distinct coverings of the $k$-simplex and the KKMS theorem of Shapley [16], where the sets in the covering are associated to faces of the $k$-simplex instead of its vertices. Further generalizations of the KKMS theorem are a polytopal version due to Komiya [12] and the colorful KKMS theorem of Shih and Lee [17].

In this note we prove a colorful polytopal KKMS theorem, extending all results above. This result is finally sufficiently general to also specialize to Bárány's celebrated colorful Carathéodory theorem [5] from 1982, which asserts that if $X_{1}, \ldots, X_{k+1}$ are subsets of $\mathbb{R}^{k}$ with $0 \in \operatorname{conv} X_{i}$ for every $i \in[k+1]$, then there exists a choice of points $x_{1} \in X_{1}, \ldots, x_{k+1} \in X_{k+1}$ such that $0 \in \operatorname{conv}\left\{x_{1}, \ldots, x_{k+1}\right\}$. Carathéodory's classical result is the case $X_{1}=X_{2}=\cdots=$ $X_{k+1}$. We deduce the colorful Carathéodory theorem from our main result in Section 3.

For a set $\sigma \subset \mathbb{R}^{k}$ we denote by $C_{\sigma}$ the cone of $\sigma$, that is, the union of all rays emanating from the origin that intersect $\sigma$. Our main result is the following: 
Theorem 1.1. Let $P$ be a $k$-dimensional polytope with $0 \in P$. Suppose for every nonempty, proper face $\sigma$ of $P$ we are given $k+1$ points $y_{\sigma}^{(1)}, \ldots, y_{\sigma}^{(k+1)} \in C_{\sigma}$ and $k+1$ closed sets $A_{\sigma}^{(1)}, \ldots, A_{\sigma}^{(k+1)} \subset P$. If $\sigma \subset \bigcup_{\tau \subset \sigma} A_{\tau}^{(j)}$ for every face $\sigma$ of $P$ and every $j \in[k+1]$, then there exist faces $\sigma_{1}, \ldots, \sigma_{k+1}$ of $P$ such that $0 \in \operatorname{conv}\left\{y_{\sigma_{1}}^{(1)}, \ldots, y_{\sigma_{k+1}}^{(k+1)}\right\}$ and $\bigcap_{i=1}^{k+1} A_{\sigma_{i}}^{(i)} \neq \emptyset$.

Our proof of this result relies on a topological mapping degree argument. As such, it is entirely different from Bárány's proof of the colorful Carathéodory theorem, and thus provides a new topological route to prove this theorem. Our argument is also less involved than the topological proof given recently by Meunier, Mulzer, Sarrabezolles, and Stein [14] to show that algorithmically finding the configuration whose existence is guaranteed by the colorful Carathéodory theorem is in PPAD (that is, informally speaking, it can be found by a path-following algorithm). Our method, however, involves a limiting argument and thus does not have immediate algorithmic consequences. Finally, our proof of Theorem 1.1 exhibits a surprisingly simple way to prove KKMS-type results and their polytopal and colorful extensions.

As an application of Theorem 1.1 we prove a bound on the piercing numbers of colorful $d$-interval hypergraphs. A $d$-interval is a union of at most $d$ disjoint closed intervals on $\mathbb{R}$. A $d$-interval $h$ is separated if it consists of $d$ disjoint interval components $h=h^{1} \cup \cdots \cup h^{d}$ with $h^{i+1} \subset(i, i+1)$ for $i \in\{0, \ldots, d-1\}$. A hypergraph of (separated) $d$-intervals is a hypergraph $H$ whose vertex set is $\mathbb{R}$ and whose edge set is a finite family of (separated) $d$-intervals.

A matching in a hypergraph $H=(V, E)$ with vertex set $V$ and edge set $E$ is a set of disjoint edges. A cover is a subset of $V$ intersecting all edges. The matching number $\nu(H)$ is the maximal size of a matching, and the covering number (or piercing number) $\tau(H)$ is the minimal size of a cover. Tardos [19] and Kaiser [10] proved the following bound on the covering number in hypergraphs of $d$-intervals:

Theorem 1.2 (Tardos [19], Kaiser [10]). In every hypergraph $H$ of d-intervals we have $\tau(H) \leq\left(d^{2}-d+1\right) \nu(H)$. Moreover, if $H$ is a hypergraph of separated d-intervals then $\tau(H) \leq\left(d^{2}-d\right) \nu(H)$.

Matoušek [13] showed that this bound is not far from the truth: There are examples of hypergraphs of $d$-intervals in which $\tau=\Omega\left(\frac{d^{2}}{\log d} \nu\right)$. Aharoni, Kaiser and Zerbib [1] gave a proof of Theorem 1.2 that used the KKMS theorem and Komiya's polytopal extension, Theorem 2.1. Using Theorem 1.1 we prove here a colorful generalization of Theorem 1.2:

Theorem 1.3. 1. Let $\mathcal{F}_{i}, i \in[k+1]$, be $k+1$ hypergraphs of d-intervals and let $\mathcal{F}=\bigcup_{i=1}^{k+1} \mathcal{F}_{i}$. If $\tau\left(\mathcal{F}_{i}\right)>k$ for all $i \in[k+1]$, then there exists a collection $\mathcal{M}$ of pairwise disjoint $d$ intervals in $\mathcal{F}$ of size $|\mathcal{M}| \geq \frac{k+1}{d^{2}-d+1}$, with $\left|\mathcal{M} \cap \mathcal{F}_{i}\right| \leq 1$.

2. Let $\mathcal{F}_{i}, i \in[k d+1]$, be $k d+1$ hypergraphs of separated d-intervals and let $\mathcal{F}=\bigcup_{i=1}^{k+1} \mathcal{F}_{i}$. If $\tau\left(\mathcal{F}_{i}\right)>k d$ for all $i \in[k+1]$, then there exists a collection $\mathcal{M}$ of pairwise disjoint separated d-intervals in $\mathcal{F}$ of size $|\mathcal{M}| \geq \frac{k+1}{d-1}$, with $\left|\mathcal{M} \cap \mathcal{F}_{i}\right| \leq 1$. 
Note that Theorem 1.2 is the case where all the hypergraphs $\mathcal{F}_{i}$ are the same. In Section 2 we introduce some notation and, as an introduction to our methods, provide a new simple proof of Komiya's theorem. Then, in Section 3, we prove Theorem 1.1 and use it to derive Bárány's colorful Carathéodory theorem. Section 4 is devoted to the proof of Theorem 1.3.

\section{Coverings of polytopes And Komiya's TheOrem}

Let $\Delta_{k}$ be the $k$-dimensional simplex with vertex set $[k+1]$ realized in $\mathbb{R}^{k+1}$ as $\left\{x \in \mathbb{R}_{\geq 0}^{k+1}\right.$ : $\left.\sum_{i=1}^{k+1} x_{i}=0\right\}$. For every $S \subset[k+1]$ let $\Delta^{S}$ be the face of $\Delta_{k}$ spanned by the vertices in $S$. Recall that the KKM theorem asserts that if $A_{1}, \ldots, A_{k+1}$ are closed sets covering $\Delta_{k}$ so that $\Delta^{S} \subset \bigcup_{i \in S} A_{i}$ for every $S \subset[k+1]$, then the intersection of all the sets $A_{i}$ is non-empty. We will refer to covers $A_{1}, \ldots, A_{k+1}$ as above as KKM cover.

A generalization of this result, known as the KKMS theorem, was proven by Shapley [16] in 1973. Now we have a cover of $\Delta_{k}$ by closed sets $A_{T}, T \subset[k+1]$, so that $\Delta^{S} \subset \bigcup_{T \subset S} A_{T}$ for every $S \subset[k+1]$. Such a collection of sets $A_{T}$ is called KKMS cover. The conclusion of the KKMS theorem is that there exists a balanced collection of $T_{1}, \ldots, T_{m}$ of subsets of $[k+1]$ for which $\bigcap_{i=1}^{m} A_{T_{i}} \neq \emptyset$. Here $T_{1}, \ldots, T_{m}$ form a balanced collection if the barycenters of the corresponding faces $\Delta_{T_{1}}, \ldots, \Delta_{T_{m}}$ contain the barycenter of $\Delta_{k}$ in their convex hull.

A different generalization of the KKM theorem is a colorful version due to Gale [9]. It states that given $k+1 \mathrm{KKM}$ covers $A_{1}^{(i)}, \ldots, A_{k+1}^{(i)}, i \in[k+1]$, of the $k$-simplex $\Delta_{k}$, there is a permutation $\pi$ of $[k+1]$ such that $\bigcap_{i \in[k+1]} A_{\pi(i)}^{(i)}$ is nonempty. This theorem is colorful in the sense that we think of each KKM cover as having a different color; the theorem then asserts that there is an intersection of $k+1$ sets of pairwise distinct colors associated to pairwise distinct vertices. Asada et al. [2] showed that one can additionally prescribe $\pi(1)$.

In 1993 Shih and Lee [17] proved a common generalization of the KKMS theorem and Gale's colorful KKM theorem: Given $k+1$ such KKMS covers $A_{T}^{i}, T \subset[k+1], i \in[k+1]$, of $\Delta_{k}$, there exists a balanced collection of $T_{1}, \ldots, T_{k+1}$ of subsets of $[k+1]$ for which we have $\bigcap_{i=1}^{m} A_{T_{i}}^{i} \neq \emptyset$.

Another far reaching extension of the KKMS theorem to general polytopes is due to Komiya [12] from 1994. Komiya proved that the simplex $\Delta_{k}$ in the KKMS theorem can be replaced by any $k$-dimensional polytope $P$, and that the barycenters of the faces can be replaced by any points $y_{\sigma}$ in the face $\sigma$ :

Theorem 2.1 (Komiya's theorem [12]). Let $P$ be a polytope, and for every nonempty face $\sigma$ of $P$ choose a point $y_{\sigma} \in \sigma$ and a closed set $A_{\sigma} \subset P$. If $\sigma \subset \bigcup_{\tau \subset \sigma} A_{\tau}$ for every face $\sigma$ of $P$, then there are faces $\sigma_{1}, \ldots, \sigma_{m}$ of $P$ such that $y_{P} \in \operatorname{conv}\left\{y_{\sigma_{1}}, \ldots, y_{\sigma_{m}}\right\}$ and $\bigcap_{i=1}^{m} A_{\sigma_{i}} \neq \emptyset$.

This specializes to the KKMS theorem if $P$ is the simplex and each point $y_{\sigma}$ is the barycenter of the face $\sigma$. Moreover, there are quantitative versions of the KKM theorem due to De Loera, Peterson, and Su [6] as well as Asada et al. [2] and KKM theorems for general pairs of spaces due to Musin [15]. 
To set the stage we will first present a simple proof of Komiya's theorem. Recall that the KKM theorem can be easily deduced from Sperner's lemma on vertex labelings of triangulations of a simplex. Our proof of Komiya's theorem - just as Shapley's original proof of the KKMS theorem - first establishes an equivalent Sperner-type version. A Sperner-Shapley labeling of a triangulation $T$ of a polytope $P$ is a map $f: V(T) \longrightarrow\{\sigma$ : $\sigma$ a nonempty face of $P\}$ from the vertex set $V(T)$ of $T$ to the set of nonempty faces of $P$ such that $f(v) \subset \operatorname{supp}(v)$, where $\operatorname{supp}(v)$ is the minimal face of $P$ containing $v$. We prove the following polytopal Sperner-Shapley theorem that will imply Theorem 2.1 by a limiting and compactness argument:

Theorem 2.2. Let $T$ be a triangulation of the polytope $P \subset \mathbb{R}^{k}$, and let $f: V(T) \longrightarrow\{\sigma$ : $\sigma$ a nonempty face of $P\}$ be a Sperner-Shapley labeling of T. For every nonempty face $\sigma$ of $P$ choose a point $y_{\sigma} \in \sigma$. Then there is a face $\tau$ of $T$ such that $y_{P} \in \operatorname{conv}\left\{y_{f(v)}: v\right.$ vertex of $\left.\tau\right\}$.

Proof. The Sperner-Shapley labeling $f$ maps vertices of the triangulation $T$ of $P$ to faces of $P$; thus mapping vertex $v$ to the chosen point $y_{f(v)}$ in the face $f(v)$ and extending linearly onto faces of $T$ defines a continuous map $F: P \longrightarrow P$. By the Sperner-Shapley condition for every face $\sigma$ of $P$ we have that $F(\sigma) \subset \sigma$. This implies that $F$ is homotopic to the identity on $\partial P$, and thus $\left.F\right|_{\partial P}$ has degree one. Then $F$ is surjective and we can find a point $x \in P$ such that $F(x)=y_{P}$. Let $\tau$ be the smallest face of $T$ containing $x$. By definition of $F$ the image $F(\tau)$ is equal to the convex hull $\operatorname{conv}\left\{y_{f(v)}: v\right.$ vertex of $\left.\tau\right\}$.

Proof of Theorem 2.1. Let $\varepsilon>0$, and let $T$ be a triangulation of $P$ such that every face of $T$ has diameter at most $\varepsilon$. Given a cover $\left\{A_{\sigma}: \sigma\right.$ a nonempty face of $\left.P\right\}$ that satisfies the covering condition of the theorem we define a Sperner-Shapley labeling in the following way: For a vertex $v$ of $T$, label $v$ by a face $\sigma \subset \operatorname{supp}(v)$ such that $v \in A_{\sigma}$. Such a face $\sigma$ exists since $v \in \operatorname{supp}(v) \subset \bigcup_{\sigma \subset \operatorname{supp}(v)} A_{\sigma}$. Thus by Theorem 2.2 there is a face $\tau$ of $T$ whose vertices are labeled by faces $\sigma_{1}, \ldots, \sigma_{m}$ of $P$ such that $y_{P} \in \operatorname{conv}\left\{y_{\sigma_{1}}, \ldots, y_{\sigma_{m}}\right\}$. In particular, the $\varepsilon$-neighborhoods of the sets $A_{\sigma_{i}}, i \in[m]$, intersect. Now let $\varepsilon$ tend to zero. As there are only finitely many collections of faces of $P$, one collection $\sigma_{1}, \ldots, \sigma_{m}$ must appear infinitely many times. By compactness of $P$ the sets $A_{\sigma_{i}}, i \in[m]$, then all intersect since they are closed.

Note that Theorem 2.1 is true also if all the sets $A_{\sigma}$ are open in $P$. Indeed, given an open cover $\left\{A_{\sigma}: \sigma\right.$ a nonempty face of $\left.P\right\}$ of $P$ as in Theorem 2.1, we can find closed sets $B_{\sigma} \subset A_{\sigma}$ that have the same nerve as $A_{\sigma}$ (namely, any collection of sets $\left\{B_{\sigma_{i}}: i \in I\right\}$ intersects if and only if the corresponding collection $\left\{A_{\sigma_{i}}: i \in I\right\}$ intersects) and still satisfy $\sigma \subset \bigcup_{\tau \subset \sigma} B_{\tau}$ for every face $\sigma$ of $P$.

\section{A colorful Komiya theorem}

Recall that the colorful KKMS theorem of Shih and Lee [17] states the following: If for every $i \in[k+1]$ the collection $\left\{A_{\sigma}^{i}: \sigma\right.$ a nonempty face of $\left.\Delta_{k}\right\}$ forms a KKMS cover of $\Delta_{k}$, then 
there exists a balanced collection of faces $\sigma_{1}, \ldots, \sigma_{k+1}$ so that $\bigcap_{i=1}^{k+1} A_{\sigma_{i}}^{i} \neq \emptyset$. Theorem 1.1, proved in this section, is a colorful extension of Theorem 2.1, and thus generalizes the colorful KKMS theorem to any polytope.

Let $P$ be a $k$-dimensional polytope. Suppose that for every nonempty face $\sigma$ of $P$ we choose $k+1$ points $y_{\sigma}^{(1)}, \ldots, y_{\sigma}^{(k+1)} \in \sigma$ and $k+1$ closed sets $A_{\sigma}^{(1)}, \ldots, A_{\sigma}^{(k+1)} \subset P$, so that $\sigma \subset \bigcup_{\tau \subset \sigma} A_{\tau}^{(j)}$ for every face $\sigma$ of $P$ and every $j \in[k+1]$. Theorem 2.1 now guarantees that for every fixed $j \in[k+1]$ there are faces $\sigma_{1}^{(j)}, \ldots, \sigma_{m_{j}}^{(j)}$ of $P$ such that $y_{P}^{(j)} \in$ $\operatorname{conv}\left\{y_{\sigma_{1}}^{(j)}, \ldots, y_{\sigma_{m_{j}}}^{(j)}\right\}$ and $\bigcap_{i=1}^{m_{j}} A_{\sigma_{i}}^{(j)}$ is nonempty. Now let us choose $y_{P}^{(1)}=y_{P}^{(2)}=\cdots=y_{P}^{(k+1)}$ and denote this point by $y_{P}$. The colorful Carathéodory theorem implies the existence of points $z_{j} \in\left\{y_{\sigma_{1}}^{(j)}, \ldots, y_{\sigma_{m_{j}}}^{(j)}\right\}, j \in[k+1]$, such that $y_{P} \in \operatorname{conv}\left\{z_{1}, \ldots, z_{k+1}\right\}$. Theorem 1.1 shows that this implication can be realized simultaneously with the existence of sets $B_{j} \in\left\{A_{\sigma_{1}}^{(j)}, \ldots, A_{\sigma_{m_{j}}}^{(j)}\right\}, j \in[k+1]$, such that $\bigcap_{j=1}^{k+1} B_{j}$ is nonempty. We prove Theorem 1.1 by applying the Sperner-Shapley version of Komiya's theorem - Theorem 2.2 - to a labeling of the barycentric subdivision of a triangulation of $P$. The same idea was used by $\mathrm{Su}$ [18] to prove a colorful Sperner's lemma. For related Sperner-type results for multiple Sperner labelings see Babson [3], Bapat [4], and Frick, Houston-Edwards, and Meunier [7].

Proof of Theorem 1.1. Let $\varepsilon>0$, and let $T$ be a triangulation of $P$ such that every face of $T$ has diameter at most $\varepsilon$. We will also assume that the chosen points $y_{\sigma}^{(1)}, \ldots, y_{\sigma}^{(k+1)}$ are contained in $\sigma$. This assumption does not restrict the generality of our proof since $0 \in$ $\operatorname{conv}\left\{x_{1}, \ldots, x_{k+1}\right\}$ for vectors $x_{1}, \ldots, x_{k+1} \in \mathbb{R}^{k}$ if and only if $0 \in \operatorname{conv}\left\{\alpha_{1} x_{1}, \ldots, \alpha_{k+1} x_{k+1}\right\}$ with arbitrary coefficients $\alpha_{i}>0$. Denote by $T^{\prime}$ the barycentric subdivision of $T$. We now define a Sperner-Shapley labeling of the vertices of $T^{\prime}$ : For $v \in V\left(T^{\prime}\right)$ let $\sigma_{v}$ be the face of $T$ so that $v$ lies at the barycenter of $\sigma_{v}$, let $\ell=\operatorname{dim} \sigma_{v}$, and let $\sigma$ be the minimal supporting face of $P$ containing $\sigma_{v}$. By the conditions of the theorem, $v$ is contained in a set $A_{\tau}^{(\ell+1)}$ where $\tau \subset \sigma$. We label $v$ by $\tau$. Thus by Theorem 2.2 there exists a face $\tau$ of $T^{\prime}$ (without loss of generality $\tau$ is a facet) whose vertices are labeled by faces $\sigma_{1}, \ldots, \sigma_{k+1}$ of $P$ such that $0 \in \operatorname{conv}\left\{y_{\sigma_{1}}^{(1)}, \ldots, y_{\sigma_{k+1}}^{(k+1)}\right\}$. In particular, the $\varepsilon$-neighborhoods of the sets $A_{\sigma_{i}}^{(i)}, i \in[k+1]$, intersect. Now use a limiting argument as before.

Note that by the same argument as before, Theorem 1.1 is true also if all the sets $A_{\sigma}^{(i)}$ are open.

For a point $x \neq 0$ in $\mathbb{R}^{k}$ let $H(x)=\left\{y \in \mathbb{R}^{k}:\langle x, y\rangle=0\right\}$ be the hyperplane perpendicular to $x$ and let $H^{+}(x)=\left\{y \in \mathbb{R}^{k}:\langle x, y\rangle \geq 0\right\}$ be the closed halfspace with boundary $H(x)$ containing $x$. Let us now show that Bárány's colorful Carathéodory theorem is a special case of Theorem 1.1.

Theorem 3.1 (Colorful Carathéodory theorem, Bárány [5]). Let $X_{1}, \ldots, X_{k+1}$ be finite subsets of $\mathbb{R}^{k}$ with $0 \in \operatorname{conv} X_{i}$ for every $i \in[k+1]$. Then there are $x_{1} \in X_{1}, \ldots, x_{k+1} \in X_{k+1}$ such that $0 \in \operatorname{conv}\left\{x_{1}, \ldots, x_{k+1}\right\}$. 
Proof. We will assume that 0 is not contained in any of the sets $X_{1}, \ldots, X_{k+1}$, for otherwise we are done. Let $P \subset \mathbb{R}^{k}$ be a polytope containing 0 in its interior, such that if points $x$ and $y$ belong to the same face of $P$ then $\langle x, y\rangle \geq 0$. For example, a sufficiently fine subdivision of any polytope that contains 0 in its interior (slightly perturbed to be a strictly convex polytope) satisfies this condition. We can assume that any ray emanating from the origin intersects each $X_{i}$ in at most one point by arbitrarily deleting any additional points from $X_{i}$. This will not affect the property that $0 \in \operatorname{conv} X_{i}$. Furthermore, we can choose $P$ in such a way that for each face $\sigma$ and $i \in[k+1]$ the intersection $C_{\sigma} \cap X_{i}$ contains at most one point.

For every $i \in[k+1]$ let $y_{P}^{(i)}=0$ and $A_{P}^{(i)}=\emptyset$. Now for each nonempty, proper face $\sigma$ of $P$ choose points $y_{\sigma}^{(i)}$ and sets $A_{\sigma}^{(i)}$ in the following way: If there exists $x \in C_{\sigma} \cap X_{i}$ then let $y_{\sigma}^{(i)}=x$ and $A_{\sigma}^{(i)}=\{y \in P:\langle y, x\rangle \geq 0\}=P \cap H^{+}(x)$; otherwise let $y_{\sigma}^{(i)}$ be some point in $\sigma$ and let $A_{\sigma}^{(i)}=\sigma$.

Suppose the statement of the theorem was incorrect; then in particular, we can slightly perturb the vertices of $P$ and those points $y_{\sigma}^{(i)}$ that were chosen arbitrarily in $\sigma$, to make sure that for any collection of points $y_{\sigma_{1}}^{(1)}, \ldots, y_{\sigma_{k+1}}^{(k+1)}$ and any subset $S$ of this collection of size at most $k, 0 \notin \operatorname{conv} S$.

Let us now check that with these definitions the conditions of Theorem 1.1 hold. Clearly, all the sets $A_{\sigma}^{(i)}$ are closed. The fact that $P$ is covered by the sets $A_{\sigma}^{(i)}$ for every fixed $i$ follows from the condition $0 \in \operatorname{conv} X_{i}$. Indeed, this condition implies that for every $p \in P$ there exists a point $x \in X_{i}$ with $\langle p, x\rangle \geq 0$, and therefore, for the face $\sigma$ of $P$ for which $x \in C_{\sigma}$ we have $p \in A_{\sigma}^{(i)}$.

Now fix a proper face $\sigma$ of $P$. We claim that $\sigma \subset A_{\sigma}^{(i)}$ for every $i$. Indeed, either $X_{i} \cap C_{\sigma}=\emptyset$ in which case $A_{\sigma}^{(i)}=\sigma$, or otherwise, pick $x \in X_{i} \cap C_{\sigma}$ and let $\lambda>0$ such that $\lambda x \in \sigma$; then for every $p \in \sigma$ we have $\langle p, \lambda x\rangle \geq 0$ by our assumption on $P$, and thus $\langle p, x\rangle \geq 0$, or equivalently $p \in A_{\sigma}^{(i)}$.

Thus by Theorem 1.1 there exist faces $\sigma_{1}, \ldots, \sigma_{k+1}$ of $P$ such that $\bigcap_{i=1}^{k+1} A_{\sigma_{i}}^{(i)} \neq \emptyset$ and $0 \in \operatorname{conv}\left\{y_{\sigma_{1}}^{(1)}, \ldots, y_{\sigma_{k+1}}^{(k+1)}\right\}$. We claim that $\bigcap_{i=1}^{k+1} A_{\sigma_{i}}^{(i)}$ can contain only the origin. Indeed, suppose that $0 \neq x_{0} \in \bigcap_{i=1}^{k+1} A_{\sigma_{i}}^{(i)}$. Fix $i \in[k+1]$. If $y_{\sigma_{i}}^{(i)} \in C_{\sigma_{i}} \cap X_{i}$, then since $x_{0} \in A_{\sigma_{i}}^{(i)}$ we have $y_{\sigma_{i}}^{(i)} \in H^{+}\left(x_{0}\right)$ by definition. Otherwise $x_{0} \in A_{\sigma_{i}}^{(i)}=\sigma_{i}$ and $y_{\sigma_{i}}^{(i)} \in \sigma_{i}$, so by our choice of $P$ we obtain again that $y_{\sigma_{i}}^{(i)} \in H^{+}\left(x_{0}\right)$. Thus all the points $y_{\sigma_{1}}^{(1)}, \ldots, y_{\sigma_{k+1}}^{(k+1)}$ are in $H^{+}\left(x_{0}\right)$. But since $0 \in \operatorname{conv}\left\{y_{\sigma_{1}}^{(1)}, \ldots, y_{\sigma_{k+1}}^{(k+1)}\right\}$ this implies that the convex hull of the points in $\left\{y_{\sigma_{1}}^{(1)}, \ldots, y_{\sigma_{k+1}}^{(k+1)}\right\} \cap H\left(x_{0}\right)$ contains the origin. Now, the dimension of $H\left(x_{0}\right)$ is $k-1$, and thus by Carathéodory's theorem there exists a set $S$ of at most $k$ of the points in $y_{\sigma_{1}}^{(1)}, \ldots, y_{\sigma_{k+1}}^{(k+1)}$ with $0 \in \operatorname{conv} S$, in contradiction to our general position assumption.

We have shown that $\bigcap_{i=1}^{k+1} A_{\sigma_{i}}^{(i)}=\{0\}$, and thus in particular, $A_{\sigma_{i}}^{(i)} \neq \sigma_{i}$ for all $i$. By our definitions, this implies $y_{\sigma_{i}}^{(i)} \in X_{i}$ for all $i$, concluding the proof of the theorem.

Remark 3.2. Note that we could have avoided the usage of Carathéodory's theorem in the proof of Theorem 3.1 by taking a more restrictive assumption on the polytope $P$, namely, 
that $\langle x, y\rangle>0$ whenever the points $x$ and $y$ belong to the same face of $P$. Therefore, in particular, Theorem 3.1 specializes to Carathéodory's theorem in the case where all the sets $X_{i}$ are the same.

\section{A COlorful $d$-Interval theorem}

Recall that a fractional matching in a hypergraph $H=(V, E)$ is a function $f: E \longrightarrow \mathbb{R}_{\geq 0}$ satisfying $\sum_{e: e \ni v} f(e) \leq 1$ for all $v \in V$. A fractional cover is a function $g: V \longrightarrow \mathbb{R}_{\geq 0}$ satisfying $\sum_{v: v \in e} g(v) \geq 1$ for all $e \in E$. The fractional matching number $\nu^{*}(H)$ is the maximum of $\sum_{e \in E} f(e)$ over all fractional matchings $f$ of $H$, and the fractional covering number $\tau^{*}(H)$ is the minimum of $\sum_{v \in V} g(v)$ over all fractional covers $g$. By linear programming duality, $\nu \leq \nu^{*}=\tau^{*} \leq \tau$. A perfect fractional matching in $H$ is a fractional matching $f$ in which $\sum_{e: v \in e} f(e)=1$ for every $v \in V$. It is a simple observation that a collection of sets $\mathcal{I} \subset 2^{[k+1]}$ is balanced if and only if the hypergraph $H=([k+1], \mathcal{I})$ has a perfect fractional matching (see e.g., [1]). The rank of a hypergraph $H=(V, E)$ is the maximal size of an edge in $H$. $H$ is $d$-partite if there exists a partition $V_{1}, \ldots, V_{d}$ of $V$ such that $\left|e \cap V_{i}\right|=1$ for every $e \in E$ and $i \in[d]$.

For the proof of Theorem 1.3 we will use the following theorem by Füredi.

Theorem 4.1 (Füredi [8]). If $H$ is a hypergraph of rank $d \geq 2$, then $\nu(H) \geq \frac{\nu^{*}(H)}{d-1+\frac{1}{d}}$. If, in addition, $H$ is d-partite, then $\nu(H) \geq \frac{\nu^{*}(H)}{d-1}$.

We will also need the following simple counting argument.

Lemma 4.2. If a hypergraph $H=(V, E)$ of rank $d$ has a perfect fractional matching, then $\nu^{*}(H) \geq \frac{|V|}{d}$.

Proof. Let $f: E \longrightarrow \mathbb{R}_{\geq 0}$ be a perfect fractional matching of $H$. Then $\sum_{v \in V} \sum_{e: v \in e} f(e)=$ $\sum_{v \in V} 1=|V|$. Since $f(e)$ was counted $|e| \leq d$ times in this equation for every edge $e \in E$, we have that $\nu^{*}(H) \geq \sum_{e \in E} f(e) \geq \frac{|V|}{d}$.

We are now ready to prove Theorem 1.3. The proof is an adaption of the methods in [1]. For the first part we need the simplex version of Theorem 1.1, which was already proven by Shih and Lee [17], while the second part requires our more general polytopal extension.

Proof of Theorem 1.3. For a point $\vec{x}=\left(x_{1}, \ldots, x_{k+1}\right) \in \Delta_{k}$ let $p_{\vec{x}}(j)=\sum_{t=1}^{j} x_{t} \in[0,1]$. Since $\mathcal{F}$ is finite, by rescaling $\mathbb{R}$ we may assume that $\mathcal{F} \subset(0,1)$. For every $T \subset[k+1]$ let $A_{T}^{i}$ be the set consisting of all $\vec{x} \in \Delta_{k}$ for which there exists a $d$-interval $f \in \mathcal{F}_{i}$ satisfying:

(a) $f \subset \bigcup_{j \in T}\left(p_{\vec{x}}(j-1), p_{\vec{x}}(j)\right)$, and

(b) $f \cap\left(p_{\vec{x}}(j-1), p_{\vec{x}}(j)\right) \neq \emptyset$ for each $j \in T$.

Note that $A_{T}^{i}=\emptyset$ whenever $|T|>d$.

Clearly, the sets $A_{T}^{i}$ are open. The assumption $\tau\left(\mathcal{F}_{i}\right)>k$ implies that for every $\vec{x}=$ $\left(x_{1}, \ldots, x_{k+1}\right) \in \Delta_{k}$, the set $P(\vec{x})=\left\{p_{\vec{x}}(j): j \in[k]\right\}$ is not a cover of $\mathcal{F}_{i}$, meaning that 
there exists $f \in \mathcal{F}_{i}$ not containing any $p_{\vec{x}}(j)$. This, in turn, means that $\vec{x} \in A_{T}^{i}$ for some $T \subseteq[k+1]$, and thus the sets $A_{T}^{i}$ form a cover of $\Delta_{k}$ for every $i \in[k+1]$.

To show that this is a KKMS cover, let $\Delta^{S}$ be a face of $\Delta_{k}$ for some $S \subset[k+1]$. If $\vec{x} \in \Delta^{S}$ then $\left(p_{\vec{x}}(j-1), p_{\vec{x}}(j)\right)=\emptyset$ for $j \notin S$, and hence it is impossible to have $f \cap\left(p_{\vec{x}}(j-1), p_{\vec{x}}(j)\right) \neq \emptyset$. Thus $\vec{x} \in A_{T}^{i}$ for some $T \subseteq S$. This proves that $\Delta^{S} \subseteq \bigcup_{T \subseteq S} A_{T}^{i}$ for all $i \in[k+1]$.

By Theorem 1.1 there exists a balanced collection of sets $\mathcal{T}=\left\{T_{1}, \ldots, T_{k+1}\right\}$ of subsets of $[k+1]$, satisfying $\bigcap_{i=1}^{k+1} A_{T_{i}}^{i} \neq \emptyset$. In particular, $\left|T_{i}\right| \leq d$ for all $i$. (Recall that we think of a collection of sets $\mathcal{I} \subset 2^{[k+1]}$ as faces of the $k$-dimensional simplex to apply the earlier geometric definition of balancedness.) Then by the observation mentioned above, the hypergraph $H=$ $([k+1], \mathcal{T})$ of rank $d$ has a perfect fractional matching, and thus by Lemma 4.2 we have $\nu^{*}(H) \geq \frac{k+1}{d}$. Therefore, by Theorem 4.1, $\nu(H) \geq \frac{\nu^{*}(H)}{d-1+\frac{1}{d}} \geq \frac{k+1}{d^{2}-d+1}$.

Let $M$ be a matching in $H$ of size $m \geq \frac{k+1}{d^{2}-d+1}$. Let $\vec{x} \in \bigcap_{i=1}^{k+1} A_{T_{i}}^{i}$. For every $i \in[k+1]$ let $f\left(T_{i}\right)$ be the $d$-interval of $\mathcal{F}_{i}$ witnessing the fact that $\vec{x} \in A_{T_{i}}^{i}$. Then the set $\mathcal{M}=\left\{f\left(T_{i}\right) \mid\right.$ $\left.T_{i} \in M\right\}$ is a matching of size $m$ in $\mathcal{F}$ with $\left|\mathcal{M} \cap \mathcal{F}_{i}\right| \leq 1$. This proves the first assertion of the theorem.

Now suppose that $\mathcal{F}_{i}$ is a hypergraph of separated $d$-intervals for all $i \in[k d+1]$. For $f \in \mathcal{F}$ let $f^{t} \subset(t-1, t)$ be the $t$-th interval component of $f$. We can assume without loss of generality that $f^{t}$ is nonempty. Let $P=\left(\Delta_{k}\right)^{d}$. For a $d$-tuple $T=\left(j_{i}, \ldots, j_{d}\right) \subset[k+1]^{d}$ let $A_{T}^{i}$ consist of all $\vec{X}=\vec{x}^{1} \times \cdots \times \vec{x}^{d} \in P$ for which there exists $f \in \mathcal{F}_{i}$ satisfying $f^{t} \subset\left(t-1+p_{\vec{x}^{t}}\left(j_{t}-1\right), t-1+p_{\vec{x}^{t}}\left(j_{t}\right)\right)$ for all $t \in[d]$.

Since $\tau\left(\mathcal{F}_{i}\right)>k d$, the points $t-1+p_{\vec{x}^{t}}(j), t \in[d], j \in[k]$, do not form a cover of $\mathcal{F}_{i}$. Therefore, by the same argument as before, the sets $A_{T}^{i}$ are open and satisfy the covering condition of Theorem 1.1. Thus, by Theorem 1.1, there exists a set $\mathcal{T}=\left\{T_{1}, \ldots, T_{k d+1}\right\}$ of $d$-tuples in $[k+1]^{d}$ containing the point $\left(\frac{1}{k+1}, \ldots, \frac{1}{k+1}\right) \times \cdots \times\left(\frac{1}{k+1}, \ldots, \frac{1}{k+1}\right) \in P$ in its convex hull and satisfying $\bigcap_{i \in[k d+1]} A_{T_{i}}^{i} \neq \emptyset$. Then the $d$-partite hypergraph $H=\left(\bigcup_{i=1}^{d} V_{i}, \mathcal{T}\right)$, where $V_{i}=[k+1]$ for all $i$, has a perfect fractional matching, and hence by Lemma 4.2 we have $\nu^{*}(H) \geq k+1$. By Theorem 4.1, this implies $\nu(H) \geq \frac{\nu^{*}(H)}{d-1} \geq \frac{k+1}{d-1}$. Now, by the same argument as before, by taking $\vec{X} \in \bigcap_{i \in[k d+1]} A_{T_{i}}^{i}$ we obtain a matching in $\mathcal{F}$ of the same size as a maximal matching in $H$, concluding the proof of the theorem.

\section{ACKNOWLEDGMENT}

We wish to thank Erel Segal-Halevi for pointing out a mistake in the statement of Theorem 1.3(2) in a previous version of this manuscript. This material is based upon work supported by the National Science Foundation under Grant No. DMS-1440140 while the authors were in residence at the Mathematical Sciences Research Institute in Berkeley, California, during the Fall 2017 semester. 


\section{REFERENCES}

1. R. Aharoni, T. Kaiser, and S. Zerbib, Fractional covers and matchings in families of weighted d-intervals, Combinatorica 37 (2017), no. 4, 555-572.

2. M. Asada, F. Frick, V. Pisharody, M. Polevy, D. Stoner, L. Tsang, and Z. Wellner, Fair division and generalizations of Sperner- and KKM-type results, SIAM J. Discrete Math. 32(1), 591-610 (2018).

3. E. Babson, Meunier conjecture, arXiv preprint arXiv:1209.0102 (2012).

4. R. B. Bapat, A constructive proof of a permutation-based generalization of Sperner's lemma, Math. Program. 44 (1989), no. 1-3, 113-120.

5. I. Bárány, A generalization of Carathéodory's theorem, Discrete Math. 40 (1982), no. 2-3, 141-152.

6. J. A. De Loera, E. Peterson, and F. E. Su, A polytopal generalization of Sperner's lemma, J. Combin. Theory, Ser. A 100 (2002), no. 1, 1-26.

7. F. Frick, K. Houston-Edwards, and F. Meunier, Achieving rental harmony with a secretive roommate, Amer. Math. Monthly, to appear.

8. Z. Füredi, Maximum degree and fractional matchings in uniform hypergraphs, Combinatorica 1 (1981), no. $2,155-162$.

9. D. Gale, Equilibrium in a discrete exchange economy with money, Int. J. Game Theory 13 (1984), no. 1, 61-64.

10. T. Kaiser, Transversals of d-intervals, Discrete Comput. Geom. 18 (1997), no. 2, 195-203.

11. B. Knaster, C. Kuratowski, and S. Mazurkiewicz, Ein Beweis des Fixpunktsatzes für n-dimensionale Simplexe, Fund. Math. 14 (1929), no. 1, 132-137.

12. H. Komiya, A simple proof of KKMS theorem, Econ. Theory 4 (1994), no. 3, 463-466.

13. J. Matoušek, Lower bounds on the transversal numbers of d-intervals, Discrete Comput. Geom. 26 (2001), no. $3,283-287$.

14. F. Meunier, W. Mulzer, P. Sarrabezolles, and Y. Stein, The rainbow at the end of the line: a PPAD formulation of the colorful Carathéodory theorem with applications, Proceedings of the Twenty-Eighth Annual ACM-SIAM Symposium on Discrete Algorithms, Society for Industrial and Applied Mathematics, 2017, pp. 1342-1351.

15. O. R. Musin, KKM type theorems with boundary conditions, J. Fixed Point Theory Appl. 19 (2017), no. 3, 2037-2049.

16. L. S. Shapley, On balanced games without side payments, Math. Program., Math. Res. Center Publ. (T. C. Hu and S. M. Robinson, eds.), vol. 30, Academic Press, New York, 1973, pp. 261-290.

17. M. Shih and S. Lee, Combinatorial formulae for multiple set-valued labellings, Math. Ann. 296 (1993), no. $1,35-61$.

18. F. E. Su, Rental harmony: Sperner's lemma in fair division, Amer. Math. Monthly 106 (1999), no. 10, 930-942.

19. G. Tardos, Transversals of 2-intervals, a topological approach, Combinatorica 15 (1995), no. 1, 123-134.

(FF) Dept. Math., Cornell University, Ithaca, NY

Email address: ff238@cornell.edu

(SZ) Dept. Math., University of Michigan, Ann Arbor, Mi

Email address: zerbib@umich.edu 